\title{
A Comparative Analysis of the Gene Expression Profiles of Small Cell Esophageal Carcinoma, Small Cell Lung Cancer, and Esophageal Adeno/Squamous Carcinoma
}

\author{
Di Liu ${ }^{1,2,3 \dagger}$, Junmiao Wen ${ }^{1,2,3 \dagger}$, Jiayan Chen ${ }^{1,2,3}$, Boyan Wang ${ }^{1,2,3}$, Xinyan $X u^{1,2,3}$, \\ Zhen Zhang ${ }^{1,2,3}$ and Min Fan ${ }^{1,2,3 *}$
}

${ }^{1}$ Department of Radiation Oncology, Fudan University Shanghai Cancer Center, Shanghai, China, ${ }^{2}$ Department of Oncology, Shanghai Medical College, Fudan University, Shanghai, China, ${ }^{3}$ Shanghai Key Laboratory of Radiation Oncology, Shanghai, China

\section{OPEN ACCESS}

Edited by:

Christopher William Seder, Rush University Medical Center,

United States

Reviewed by:

Zhentao Yu,

Tianjin Medical University Cancer

Institute and Hospital, China

Long-Qi Chen,

Sichuan University, China

*Correspondence:

Min Fan

fanmin_fuscc@aliyun.com

these authors have contributed equally to this work

Specialty section:

This article was submitted to Thoracic Surgery,

a section of the journal

Frontiers in Surgery

Received: 18 January 2021 Accepted: 08 June 2021 Published: 30 July 2021

Citation:

Liu D, Wen J, Chen J, Wang B, Xu X, Zhang Z and Fan M (2021) A Comparative Analysis of the Gene Expression Profiles of Small Cell

Esophageal Carcinoma, Small Cell Lung Cancer, and Esophageal Adeno/Squamous Carcinoma.

Front. Surg. 8:655159.

doi: 10.3389/fsurg.2021.655159
Purpose/objectives: Primary small cell esophageal carcinoma (SCEC) is a rare malignancy without an established treatment strategy. This study investigated the gene expression profile of SCEC and compared it with the expression profiles of small cell lung cancer (SCLC) and esophageal adeno/squamous carcinoma (EAC/ESCC).

Materials/methods: All patients with SCEC, SCLC, and EAC/ESCC in the Surveillance, Epidemiology, and End Results (SEER) database 1973-2014 were included. Overall survival (OS) and prognostic analysis were conducted. De novo expression array analysis was performed on three pairs of frozen primary SCEC tissues and the corresponding normal samples from the institutional tissue bank using the Affymetrix HG U133 plus 2.0 Array. These data were complemented with public domain expression data sets from the Gene Expression Omnibus (GEO) repository using the same working platforms, which included primary SCLC, EAC/ESCC, and normal lung/esophagus specimens (series GSE30219 and GSE26886). After individual normalization, the primary tumors were submitted to statistical analysis (GeneSpring GX 13.0) to identify the differentially expressed genes (DEGs) relative to their paired normal tissues. Enrichments of genes categorized by function and gene interactions were analyzed by DAVID 6.8 and STRING 11.0, respectively.

Results: The clinical outcomes of the patients with SCEC were significantly more worse than those with EAC/ESCC and SCLC in the SEER database. SCEC had more DEGs in common with SCLC than EAC/ESCC [829 vs. 450; false discovery rate (FDR) $<0.01$; and fold change $\geq 2$ ], leading to a stronger correlation between SCEC and SCLC (Pearson's correlation coefficient was 0.60 for SCEC vs. SCLC, 0.51 or 0.45 for SCEC vs. ESCC or EAC, and the coefficient was 0.73 for ESCC vs. EAC). Similar findings were obtained by principal component analysis (PCA) using all DEGs retrieved from these four groups. Functional annotation showed that a higher proportion of pathways and biological processes were common between SCEC and SCLC and were associated with the cell cycle (mitosis), DNA replication, telomere maintenance, DNA repair, and P53 
and RB pathways (Benjamini $p<0.05$ ). Compared with EAC/ESCC, SCEC shared more co-upregulated DEGs coding for the aforementioned common pathways with SCLC (584 vs. 155). In addition, SCEC and SCLC were found to have possessed overlapping gene-interactive networks, with centromere protein $F(C E N P F)$, never in mitosis gene A-related kinase 2 (NEK2), kinesin family member 11 (KIF11), thymopoietin (TMPO), and forkhead box protein M1 (FOXM1) as common skeletons centered by gene regulatory network (NUF2).

Conclusions: This study is the first attempt to examine the genomic signatures of SCEC at the transcriptomic level and compare the expression profiles between SCEC, SCLC, and EAC/ESCC. Our preliminary data indicate that SCEC and SCLC display notably similar patterns of gene expression for mitosis and DNA repair. Further validation studies are warranted.

Keywords: small cell esophageal carcinoma, small cell lung cancer, esophageal squamous carcinoma, gene expression profile, esophageal adenocarcinoma

\section{INTRODUCTION}

Small cell carcinoma (SCC) is a highly aggressive malignancy that predominantly arises in the lung. Primary small cell esophageal carcinoma (SCEC) is the most common extrapulmonary SCC $(\sim 2 \%)$, with a reported incidence rate of $0.05-3.1 \%$ among all esophageal neoplasms (1-3). Due to a lack of prospective clinical trials or cell line experimental data, a consensus on treatment strategies for patients with SCEC has not been reached $(4,5)$. Previous studies have indicated similarities in pathology and clinical manifestations between SCEC and small cell lung cancer (SCLC), and patients with SCEC are staged and treated following the well-established therapeutic strategies for SCLC $(4,6)$ However, patients with SCEC have a significantly worse prognosis than those with esophageal adeno/squamous carcinoma (EAC/ESCC) and SCLC. Generally, patients with SCEC die within 2 years of diagnosis and experience a median survival of only $8-13$ months. Chemotherapy is initially effective for SCEC, but most patients suffer a rapid recurrence and respond inadequately to second-line chemotherapy. More effective and precise therapeutic strategies for SCEC are urgently needed (7-9).

The lung and esophagus arise from the anterior foregut endoderm in the thorax, and they share common properties during development $(10,11)$. Theoretically, on one hand, SCCs in the lung and esophagus may be more similar than those occurring in other organs. On the other hand, the tissue of origin of a tumor is just as important as the mutations that drive it. The tissue of origin is an important determinant of how a tumor meets its metabolic needs (12). Thus, it seems essential to analyze the molecular characteristics of SCEC and identify the differences between SCLC and EAC/ESCC.

Although the genetic landscape of SCLC and EAC/ESCC has been extensively studied, little is known about SCEC (1315). Gene expression profiling can investigate altered cellular mechanisms, thus improving our understanding of various diseases and enabling the development of novel therapeutic targets (16). SCEC, SCLC, and EAC/ESCC are highly aggressive cancers, but their detailed differences on the transcriptional levels are currently unknown. To the best of our knowledge, comparative analyses of gene expression profiles of these malignancies have not been reported so far, which is the starting point of this study.

In this study, we compared the overall survival (OS) data of SCEC, SCLC, and EAC/ESCC from the Surveillance, Epidemiology, and End Results (SEER) database. Then, genes with significantly altered expression in SCEC were screened and identified. We compared the gene expression profile of SCEC with the known data of SCLC and EAC/ESCC to highlight biomolecular markers with potential clinical significance. Finally, quantitative reverse transcription (qRT)-PCR analysis was performed to confirm the differential expression of 10 of these genes.

\section{MATERIALS AND METHODS}

\section{Patient Collection}

This study utilized the SEER-18 registry databases, which currently cover $28 \%$ of the population of the United States. SEER routinely collects demographic, tumor site, stage at diagnosis, the first course of treatment, and follow-up of vital status data. We retrieved data from 1973 to 2014 using SEER 8.3.5 software and searched for all cases of SCEC using the ICDO-3 codes 8041, 8043, and the primary site codes C150159. In addition, patients who were diagnosed with other subtypes of esophageal neoplasms during the same period were also identified according to the corresponding ICD-O3 codes (adenocarcinoma: 8050, 8140-8147, 8160-8162, 8180$8221,8250-8507,8514,8520-8551,8560,8570-8574,8576$, and 8940-8941; squamous cell carcinoma: 8070-8078, 8083, and 8084). Patients with SCLC were identified using the primary site codes C340-349 and the ICD-O-3 codes 8041 and 8043 . Patients were deemed eligible if they were $\geq 18$ years old, had more than 1 month of follow-up time, and the first primary tumor. 
TABLE 1 | Basic characteristics of SCEC, ESCC, EAC, and SCLC in the SEER database.

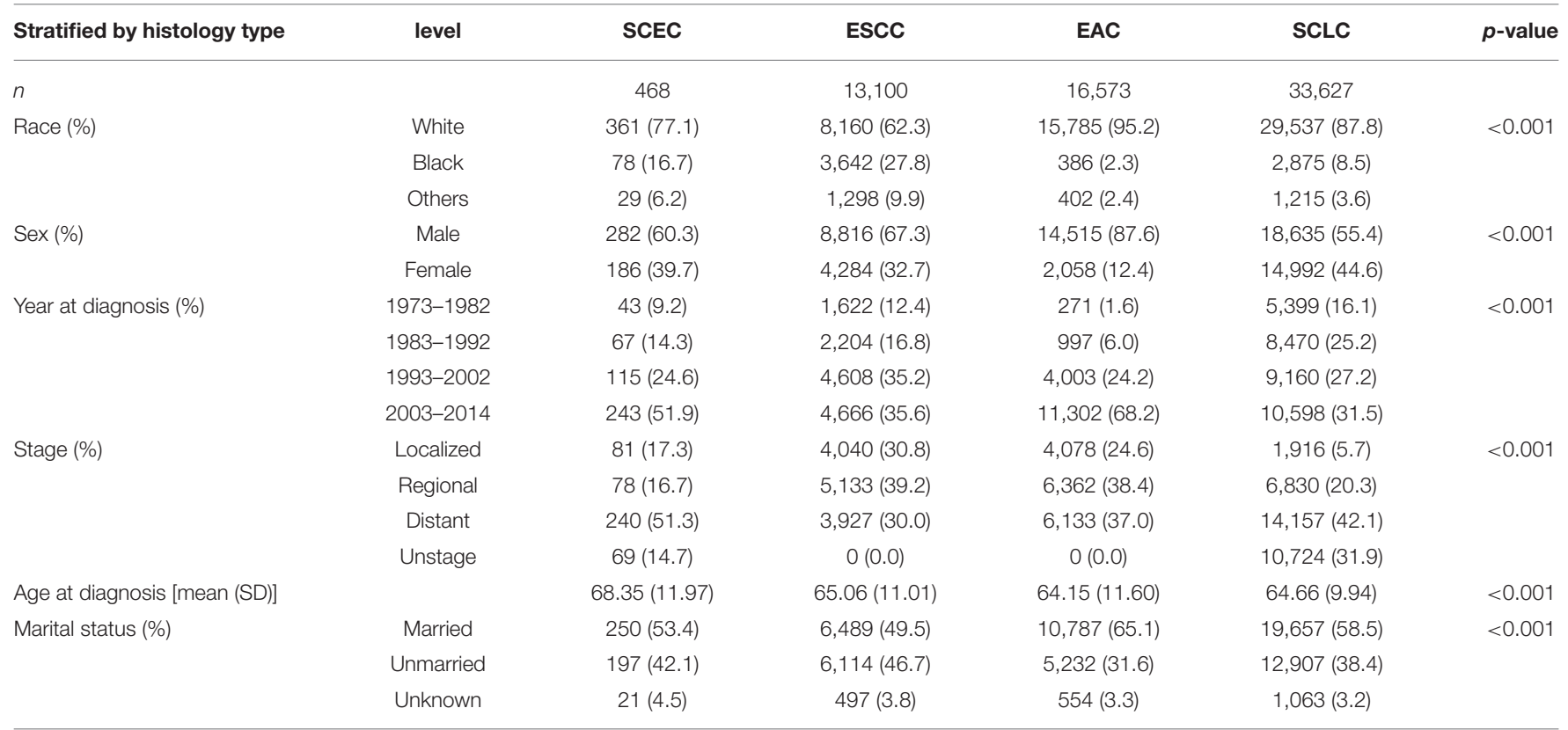

SEER, Surveillance, Epidemiology, and End Results; SCEC, small cell esophageal carcinoma; ESCC, esophageal squamous carcinoma; EAC, esophageal adenocarcinoma; SCLC, small cell lung cancer; SD, standard deviation.

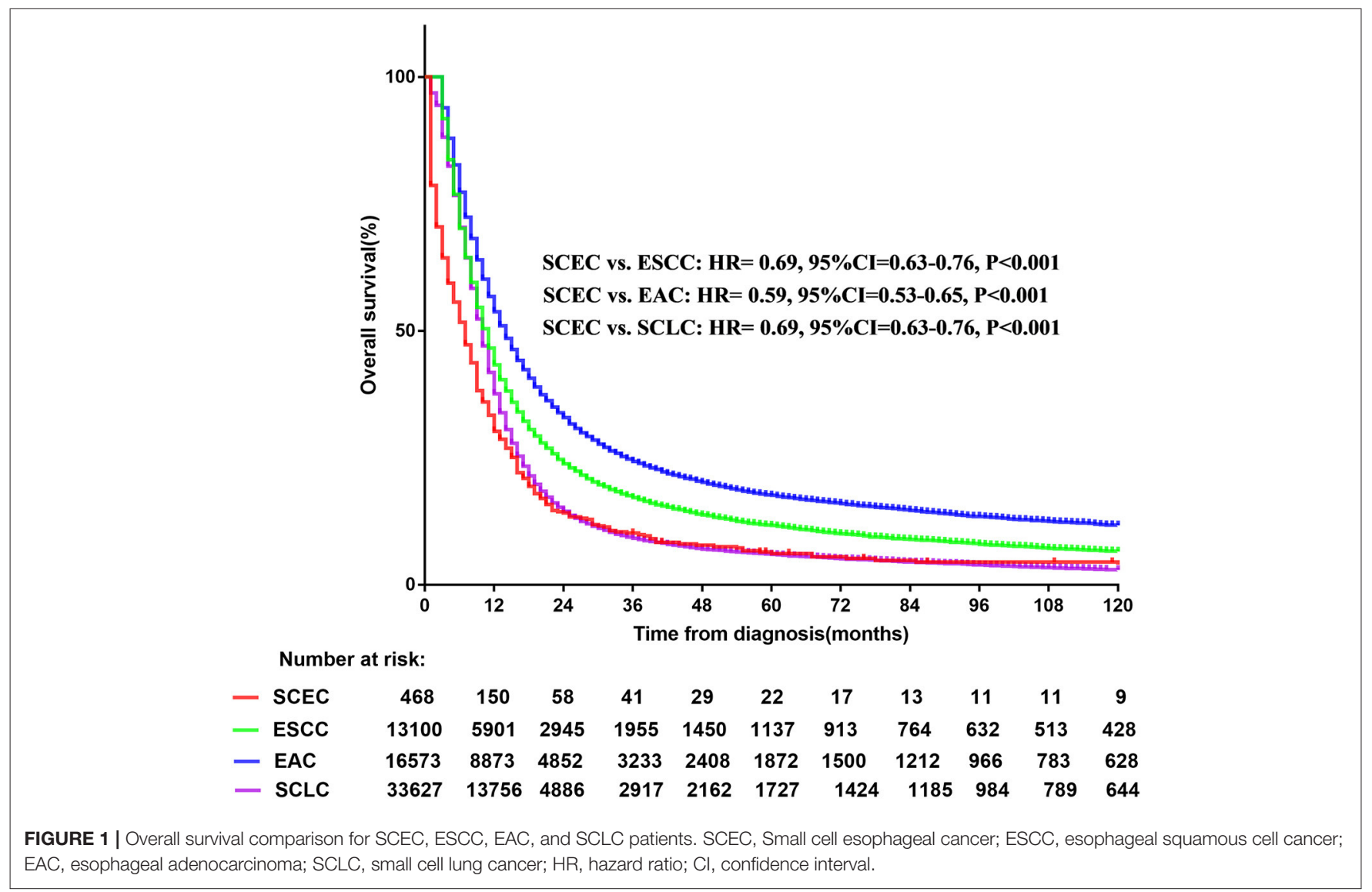




\section{Statistical Analysis}

Our analysis included age at diagnosis, sex, race, SEER summary stage, marital status, months of survival, and vital status. A log-rank test was conducted to compare the KaplanMeier survival curves. Overall survival (OS) was measured from the date of the initial treatment to the date of death or the last day of follow-up. Multivariate analyses with the Cox proportional hazards model were performed to evaluate the covariate effect on OS. Hazard ratios with 95\% CIs were employed to quantify the strength of the association between the predictors and survival. A twotailed $p$-value of $<0.05$ was considered statistically significant. All statistical calculations using were performed $\mathrm{R}$ software version 3.4.2 (Institute for Statistics and Mathematics, Vienna, Austria; www.r-project.org).

\section{Tissues and Total RNA Preparation}

A total of three SCEC tissues and matched adjacent noncancerous tissues were dissected from the surgical specimens and reviewed by at least two independent expert pathologists, and the diagnosis of SCEC was confirmed by $\mathrm{H} \& \mathrm{E}$ staining and immunohistochemistry (IHC) for synaptophysin, chromogranin A, neuro-specific enolase (NSE), neural cell adhesion molecule (CD56), and antigen KI-67 (Ki67). Any sample with squamous or adenocarcinoma differentiation was excluded. These tumor samples were pathologically assessed to have a purity of at least $60 \%$ and minimal necrosis. Additionally, by pathological assessment adjacent non-tumorigenic tissue was confirmed to be free of tumor contaminants. The selected patients did not receive any anticancer therapy before surgery and had not been diagnosed with any other cancer. Ethics approval for this study

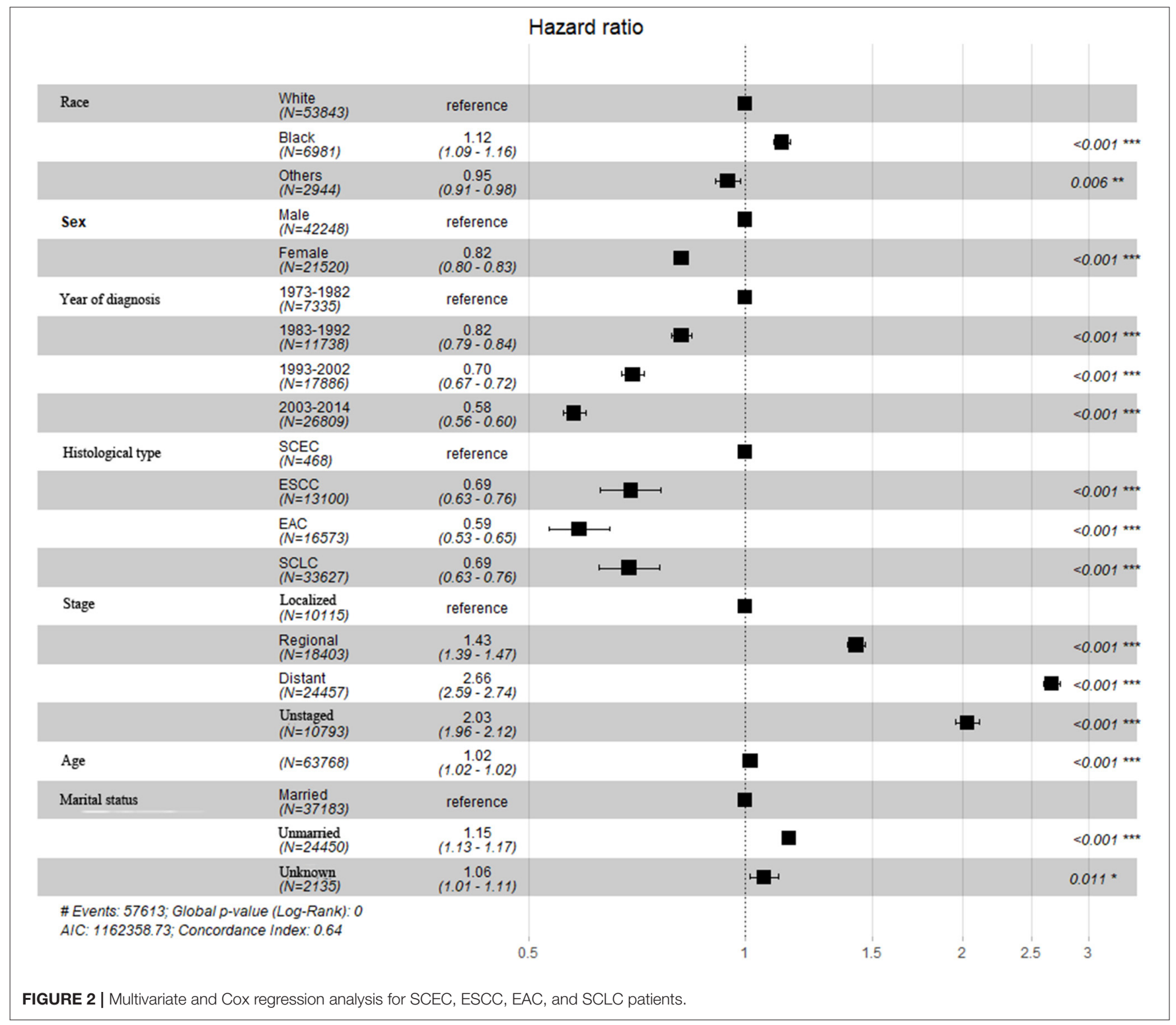


was granted by the Human Research Ethics Committee of Fudan University Shanghai Cancer Center (FUSCC), and informed consent was obtained from all patients. Two of the patients were women, and the patients had a median age of 59 years (range from 56 to 67). The primary location of all of the tumors was the middle thoracic region of the esophagus and was stage III (TNM staging system of the American Joint Committee on Cancer, 6th edition) or limited stage (Veteran's Administration Lung Cancer Study Group, VALSG). All of the patients were deceased at the last follow-up.

Total RNA was extracted from the SCEC and matched adjacent non-cancerous tissues with TRIzol reagent (Life Technologies, Carlsbad, CA, USA) according to the instructions of the manufacturer. The concentration and purity of the RNA in each sample were determined by measuring the absorbance at 260 and $280 \mathrm{~nm}$. RNA integrity was confirmed by electrophoresis on $1 \%$ agarose gels. Only RNA samples with a renewable identification number $(\mathrm{RIN})>7.5$ were applied in later microarray and quantitative reverse transcription (qRT) -PCR experiments.

\section{Gene Expression Microarray and Interactive Analysis}

The generation of cDNA and cRNA, hybridization with Affymetrix HG U133 Plus 2.0 Array (Affymetrix, Santa Clara, CA), scanning, and microarray gene expression data analyses were performed as previously described (4). These data were complemented with public domain expression data sets from the GEO repository using the same platforms, which included primary SCLC, primary EAC/ESCC, and normal lung/esophagus specimens (series GSE30219 and GSE26886). The quality control of the samples was assessed by boxplots and principal component analysis (PCA) (Supplementary Figure 1). A pairwise comparison was performed by direct comparison of differentially expressed genes (DEGs) filtered from the above four paired groups (SCEC, SCLC, EAC, and ESCC), starting from the raw data (CEL files), after individual normalization within each

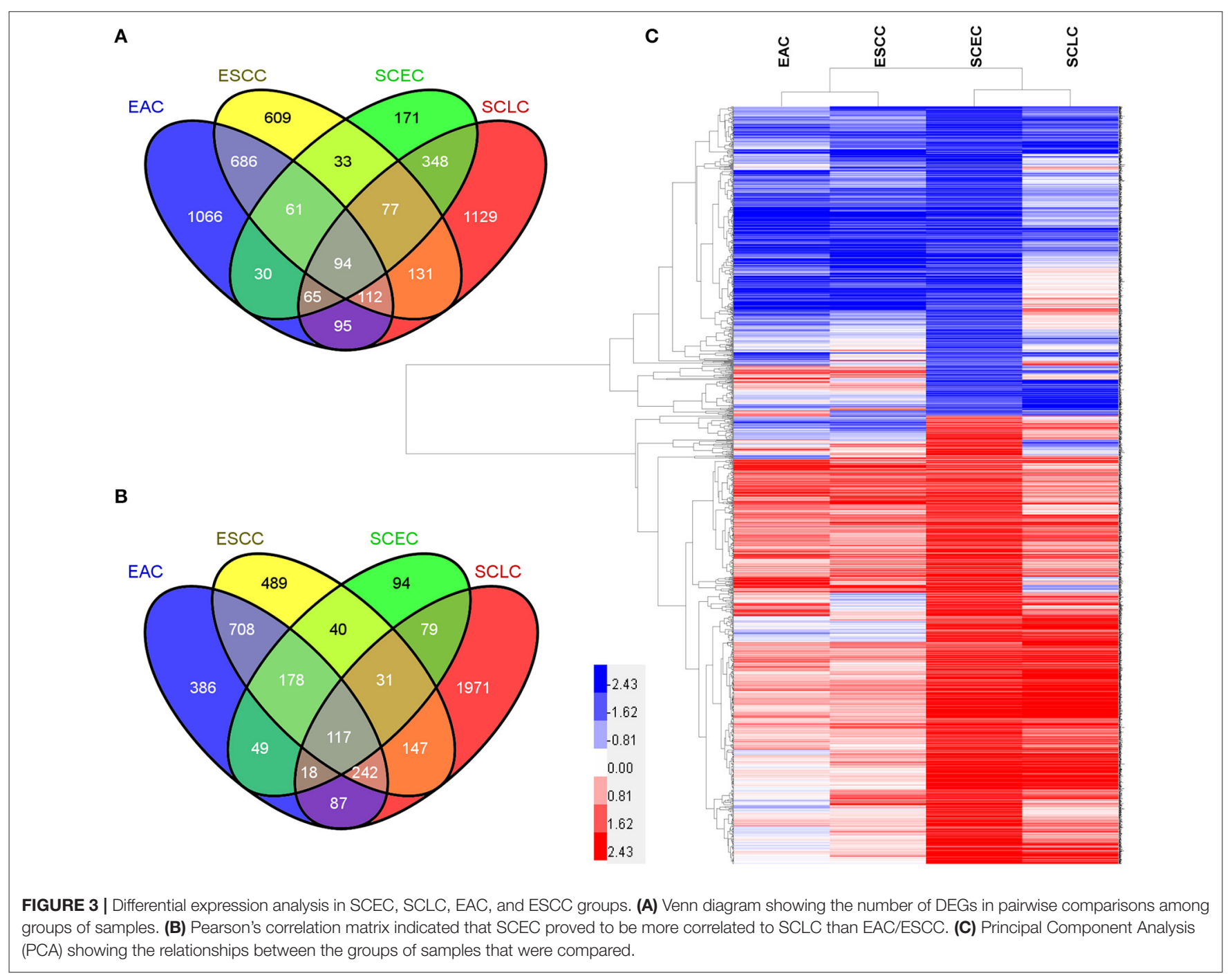


paired group and applying the same analytical approach using GeneSpring GX 13.0 software. The DEGs were analyzed through moderated $t$-test analysis with Benjamini-Hochberg multiple testing correction using the following parameters: fold change $(F C) \geq 2$ and false discovery rate (FDR) cutoff $<0.01$. The DEGs were visualized in a volcano plot (Supplementary Figure 2). Gene enrichments with functional annotation and gene interaction networks were analyzed by DAVID 6.8 and STRING 11.0 , respectively.

\section{Validation of Microarray by qRT-PCR}

Ten genes differentially expressed in SCEC compared wit $h$ matched adjacent non-cancerous tissues identified in the microarray experiment were selected for validation by qRTPCR. Total RNA extraction, cDNA synthesis, and quantification of gene expression levels were performed on a 7,500 Fast Real-Time PCR cycler (Applied Biosystems, Foster City, CA) with SYBR Green reagents (Takara Bio Inc, Shiga, Japan). as previously described (4). Primers were designed and synthesized by BioTNT Co. (Shanghai, China), and their sequences are listed in Supplementary Table 1. $\beta$-actin was used as an endogenous control. PCR reactions of each sample were conducted in triplicate. The relative expression of the target genes was calculated by $2^{-\Delta \Delta \mathrm{Ct}}$.

\section{RESULTS}

\section{SEER Data of SCEC as Compared to SCLC and EAC/ESCC}

Surveillance, Epidemiology, and End Results data of SCEC as compared to SCLC and EAC/ESCC.

A total of 63,768 patients diagnosed from 1973 to 2014 were identified from the SEER database. Among them, patients with SCLC accounted for the largest proportion $(33,627,52.7 \%)$, followed by $\operatorname{EAC}(16,573,26.0 \%)$, ESCC $(13,100,20.5 \%)$, and SCEC $(468,0.7 \%)$. The baseline characteristics are summarized in Table 1. Kaplan-Meier analyses and log-rank testing were conducted to compare the OS among these specific histological types, and the results are shown in Figure 1. Regarding OS, the 5year survival for patients with SCEC was $6.1 \%$, similar to that of patients with SCLC (5.9\%). Patients with EAC (5-year OS: 17.6\%) and ESCC (5-year OS: 11.6\%) had a better prognosis than those with the other two types. To further refine the analysis on the prognostic value of histological types, we utilized Cox models
A

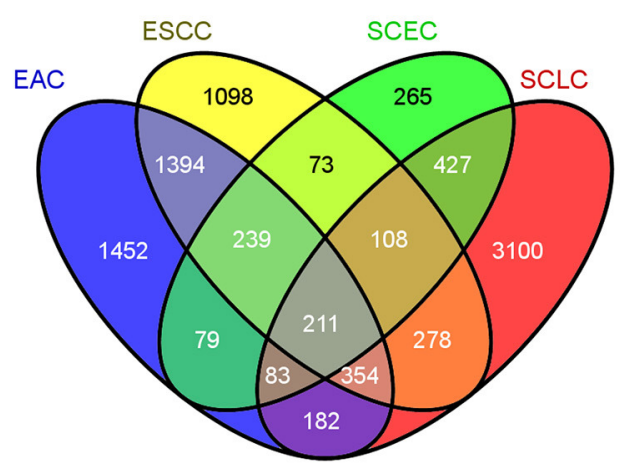

B

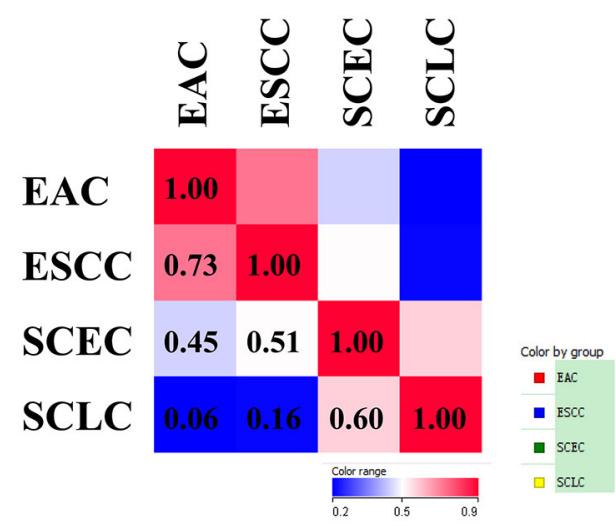

C
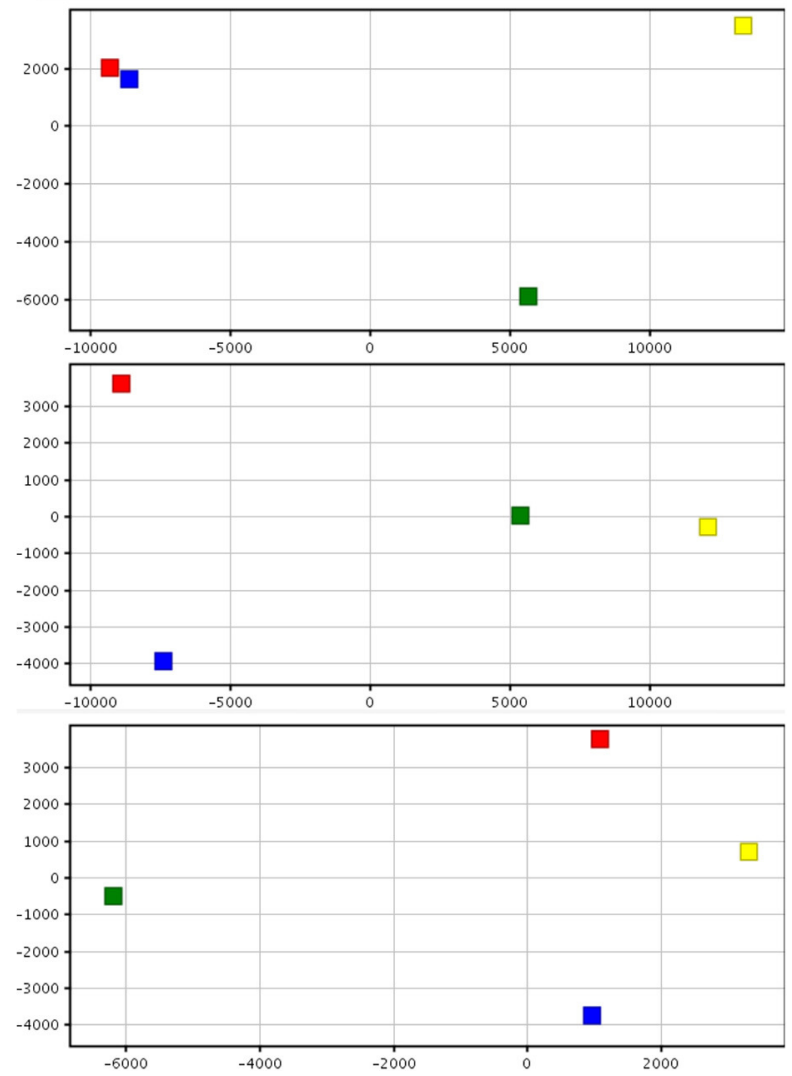

FIGURE 4 | Differential expression analysis in SCEC, SCLC, EAC, and ESCC groups divided by up-regulated and down-regulated genes. (A,B) Venn diagram showing SCEC shared more co-up regulated DEGs with SCLC compared with EAC/ESCC. (C) Hierarchical clustering of SCEC, SCLC, EAC, and ESCC groups. The color scale represents the level of expression from low (blue) to high (red). 
TABLE 2 | DAVID annotation of DEGs in SCEC group.

\begin{tabular}{|c|c|c|c|}
\hline Database & Name & Count $^{\mathrm{a}}$ & $\begin{array}{c}\text { Benjamini } \\
p \text {-value }\end{array}$ \\
\hline \multirow[t]{6}{*}{ KEGG } & DNA replication ${ }^{\mathrm{b}}$ & 19 & 8.88E-10 \\
\hline & Cell cycle & 33 & 8.61E-09 \\
\hline & P53 signaling pathway & 19 & 4.80E-05 \\
\hline & Progesterone-mediated oocyte maturation & 18 & 0.00457 \\
\hline & Base excision repair & 11 & 0.00419 \\
\hline & Oocyte meiosis & 20 & 0.00790 \\
\hline \multirow[t]{5}{*}{ REACTOME } & Cell cycle, mitotic & 100 & $3.47 \mathrm{E}-36$ \\
\hline & DNA replication & 29 & 7.19E-08 \\
\hline & DNA repair & 22 & 0.00173 \\
\hline & Cell cycle checkpoints & 23 & 0.00184 \\
\hline & Telomere maintenance & 14 & 0.00713 \\
\hline PANTHER & P53 pathway & 22 & 0.0456 \\
\hline \multirow[t]{10}{*}{ GO BP (TOP10) } & M phase & 99 & $3.91 E-28$ \\
\hline & M phase of mitotic cell cycle & 78 & $1.97 \mathrm{E}-26$ \\
\hline & Mitosis & 77 & $2.12 \mathrm{E}-26$ \\
\hline & DNA replication & 61 & $2.41 \mathrm{E}-18$ \\
\hline & DNA metabolic process & 98 & $2.54 \mathrm{E}-13$ \\
\hline & Mitotic sister chromatid segregation & 18 & 1.73E-07 \\
\hline & Spindle organization & 20 & $1.55 \mathrm{E}-07$ \\
\hline & Cell cycle checkpoint & 27 & 2.09E-06 \\
\hline & Regulation of cell cycle process & 28 & 6.97E-05 \\
\hline & DNA repair & 50 & 7.34E-05 \\
\hline \multirow[t]{3}{*}{ GO MF } & Pyrophosphatase activity & 91 & 0.00138 \\
\hline & Adenyl ribonucleotide binding & 152 & 0.00573 \\
\hline & Guanyl ribonucleotide binding & 44 & 0.0486 \\
\hline
\end{tabular}

aThreshold values: count $\geq 10$ and Benjamini p-value $<0.01$.

${ }^{b}$ The biological processes or pathways in common between SCEC and SCLC were in bold.

GO, gene ontology; BP, biological process; MF,molecular function.

to predict OS incorporating age at diagnosis, sex, ethnicity, year of diagnosis, SEER summary stage, and marital status and found that the prognosis of patients with SCEC was significantly inferior to that of the other three histological types $(p<0.001$, Figure 2).

\section{Gene Expression Profile of SCEC Compared to SCLC and EAC/ESCC}

A total of 1,485 DEGs in SCEC vs. adjacent non-cancerous tissues with 879 upregulated genes and 606 downregulated genes were identified in a previous study; these were enriched for overexpression of proliferation-associated and neuroendocrineassociated genes (4). Pathway analysis showed enrichment of DNA replication, cell cycle, mitosis, telomere maintenance, DNA repair, and p53 and RB pathways by the database for annotation, visualization, and integrated discovery (DAVID) annotation (count $\geq 10$ and Benjamini $p$-value $<0.01$ ).

The expression data demonstrated that SCEC had more DEGs in common with SCLC than EAC/ESCC ( 829 vs. 450; FDR $<0.01$; and $\mathrm{FC} \geq 2$; Figure 3 ), leading to a stronger correlation between SCEC and SCLC (Pearson's correlation coefficient was 0.60 for
SCEC vs. SCLC, 0.51 or 0.45 for SCEC vs. ESCC or EAC, and 0.73 for ESCC vs. EAC). Similar findings were obtained by PCA using all DEGs retrieved from these four groups (Figure 4). Functional annotation showed that a higher proportion of biological processes or pathways were shared in common between SCEC and SCLC and were associated with the cell cycle, mitosis, DNA replication, telomere maintenance, DNA repair, and $\mathrm{p} 53$ and $\mathrm{RB}$ pathways (count $\geq 10$ and Benjamini $p$-value $<0.05$; Table 2 and Supplementary Tables 2-4). Compared with EAC/ESCC, SCEC shared more co-upregulated DEGs coding for the aforementioned common pathways with SCLC (584 vs. 155; Figure 3). Hierarchical clustering of SCEC, SCLC, and EAC/ESCC according to gene ontology (GO) annotation is shown in Supplementary Figure 3. In addition, SCEC and SCLC possessed overlapping gene-interactive network with CENPF, NEK2, KIF11, TMPO, and FOXM1 as common skeletons centered by NUF2 (Supplementary Figure 4). The genes involved in the SCEC-regulated network were related to cell cycle, mitosis, cell cycle checkpoint, spindle organization, microtubule binding, cytoskeletal protein binding, and other biological processes (Supplementary Tables 6, 7).

\section{Validation of Microarray Results by qRT-PCR}

Genes of interest identified by microarray were validated by qRTPCR. The genes assayed were neuroendocrine-associated genes (INSM1, ASCL1, NRCAM, and SNAP25), one gene centered in the gene regulatory network (NUF2), and five possibly cancerassociated genes (PTP4A3, RFC4, REST, APEH, and FBLN2). The microarray and the qRT-PCR results demonstrated that INSM1, ASCL1, NRCAM, SNAP25, NUF2, PTP4A3, and RFC4 were significantly upregulated while REST, $A P E H$, and FBLN2 were downregulated (Figure 5).

\section{DISCUSSION}

Small cell carcinoma is a high-grade neoplasm characterized by markers of neuroendocrine differentiation and aggressive histological features (high mitotic rate, extensive necrosis, and nuclear atypia), which confers a poor clinical prognosis $(5,17$, 18). The majority of SCCs originate within the lung followed by the esophagus $(3,19-21)$. SCEC is a very rare disease with a tendency to metastasize early through lymph and blood circulation, and many recommendations about the treatment approach to SCEC are extrapolated from research on SCLC. Treatments for SCEC include surgical resection, chemotherapy, radiotherapy, and combinations of these treatments. First-line systemic chemotherapy with a platinum agent (cisplatin or carboplatin) and etoposide is recommended for most patients; however, response durations are often short, and long-time survivors are rare (22-24). Therefore, SCLC treatments are not sufficient or optimal for patients with SCEC. In addition, SCC originating in different organs may be distinct, as suggested in the literature study (20).

This study compared the survival data in the SEER database. Kaplan-Meier analysis showed that patients with SCEC had the worse OS, which was closer to SCLC and far worse than patients with EAC/ESCC. The multivariate 


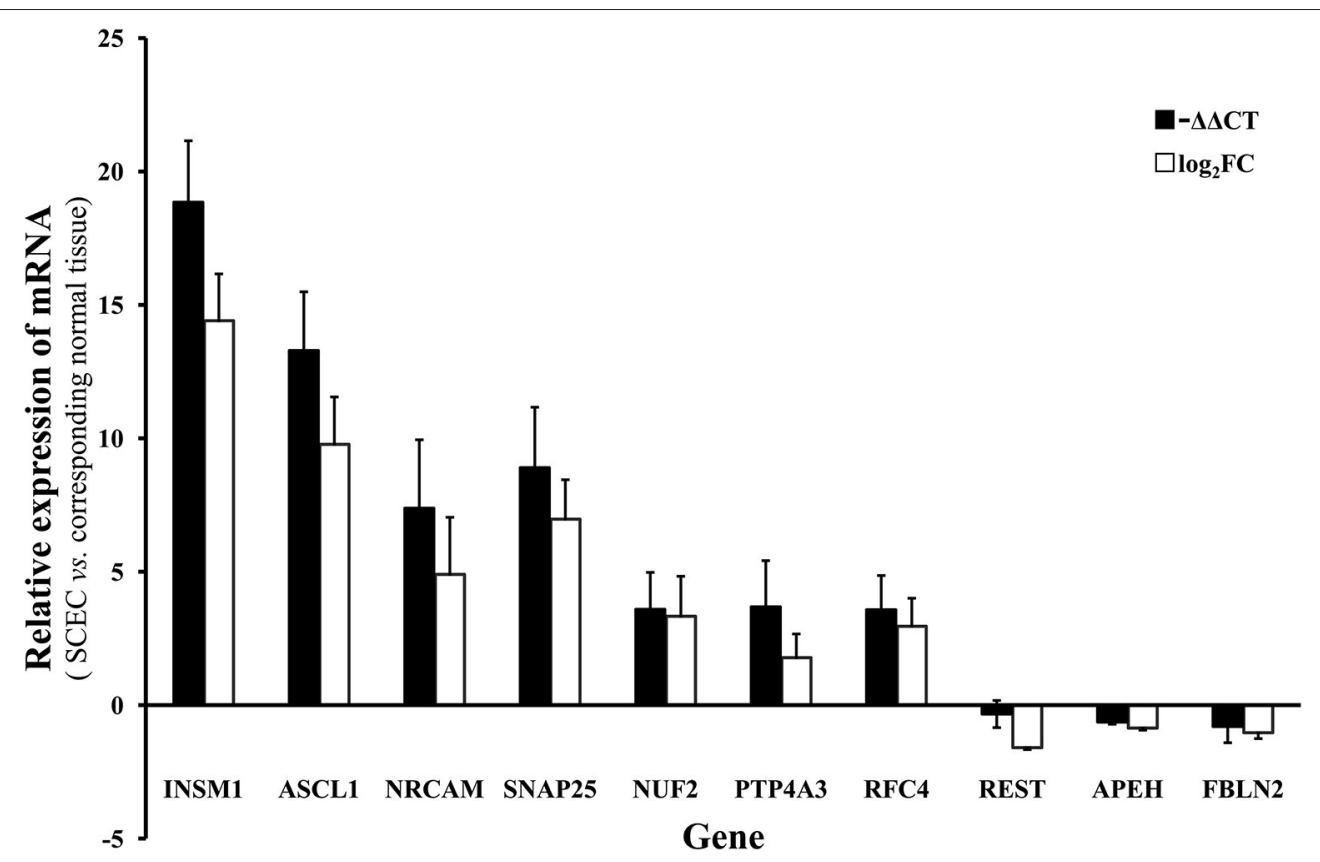

FIGURE 5 | The mRNA level of of INSM1, ASCL1, NRCAM, SNAP25, NUF2, PTP4A3, RFC4, REST, APEH, and FBLN2 in SCEC. Expression levels in SCEC were compared with the corresponding normal tissues. The $X$ axis display gene symbols and the $Y$ axis shows gene expression log ratios from microarray or qRT-PCR. Bars: standard error (SE).

analysis demonstrated that SCC was associated with a poor prognosis compared with pathological subtypes of squamous cell carcinoma or adenocarcinoma. Previous limited retrospective studies have suggested that SCEC is more malignant than other types of esophageal cancers (5). This study is in accordance with the literature study and is the first real-world study comparing the prognosis among SCEC with SCLC and EAC/ESCC using data from a large dataset.

The histogenesis of SCEC is controversial, and no definite conclusions have been made. It is assumed that SCEC may arise from amine precursor uptake and decarboxylation (APUD) cells or multipotent reserve cells $(25,26)$. SCEC and SCLC share several histological features, which support the theory that SCC arises from APUD cells. Observations of heterogeneous carcinoma components, including EAC/ESCC or mucoepidermoid carcinoma and ESCC in situ, provide evidence of derivation from multipotent reserve cells (27). It is interesting to elucidate the relationship of SCEC with SCLC and EAC/ESCC.

Genome sequencing studies have revealed several potential driver events in two other major subtypes of esophageal carcinoma and showed that they have distinct molecular characteristics, indicating the heterogeneity of esophageal carcinomas (13). A recently published SCEC landscape revealed the characteristics of the SCEC mutation spectrum and copy number variation spectrum, indicating that SCEC is highly distinct and may have a special genetic background (7). To date, detailed whole genetic studies of this disease at the mRNA level have been sparse.

In this study, we performed gene expression profiling of three patients with SCEC compared with matched adjacent non-cancerous tissues by microarray analysis. This study found that phosphatase and tensin homolog (PTEN)-, retinoblastoma protein $(R B)$-, and wingless and int-1 (WNT)-related gene sets and neuroendocrine- and proliferation-associated genes were significantly upregulated, while notch homolog 1 (NOTCH)related gene sets were downregulated in SCEC, as previously described (4). Combined with the genomic aberrance of SCEC as reported previously $(4,7)$, the aforementioned gene sets and pathways might contribute to tumorigenesis and the development of SCEC, and these results were also in line with a recent publication (21).

Furthermore, we compared the gene expression profiles of SCEC between SCLC and EAC/ESCC. Our data demonstrated that there are more gene expression similarities between SCEC and SCLC than there are between SCEC and EAC/ESCC. We observed that DEGs in SCEC were significantly enriched in the cell cycle, mitosis, DNA replication, telomere maintenance, DNA repair, and p53 and $\mathrm{RB}$ pathways, which is highly concordant with those in SCLC. In addition, SCEC and SCLC display notably similar patterns of gene-interactive networks with CENPF, NEK2, KIF11, TMPO, and FOXM1 as common skeletons centered by NUF2. In terms of the gene expression profile, the characteristics of SCEC are unique but more closely resembled SCLC than EAC/ESCC, as they share similar signaling pathways and gene-interactive networks. This similarity of expression profiles between SCEC and SCLC is consistent with the poor prognosis of SCC, since SCEC and SCLC are both highly aggressive.

With the deepening research studies into tumor biology, SCLC has entered the era of precision medicine (28). SCEC still remains outside the realm of precision medicine, where 
chemotherapy is the bedrock of treatment. Without biomarkers predictive of efficacy and toxicity and in the absence of precise identification of optimal treatment strategies, the prognosis of patients with SCEC is dismal. In addition, as our data suggested in the study, SCEC is a highly heterogeneous disease; however, its heterogeneous biology is poorly understood. Our attempt is only the first step, which has enabled a more comprehensive understanding of the transcriptomic landscape of SCEC.

A large-scale study is needed because our study had many limitations, such as a small number of samples and difficulty examining the protein level of interesting DEGs from microarrays. Only a small proportion of SCEC are resectable; inevitably, small numbers of samples are available. SCEC is a rare and deadly cancer. Although, we only examined insufficient cases, this study has added to the knowledge of SCEC at the transcriptomic level and highlights the potential useful genes and pathways for more precise diagnosis and treatment. Further, investigations based on the large-scale collection of samples are needed.

\section{CONCLUSIONS}

This study is the first to examine the genomic signatures of SCEC from a gene expression perspective with comparison to SCLC and EAC/ESCC. SCEC has an extremely poor prognosis compared with SCLC and EAC/ESCC. Our preliminary data indicated that SCEC is a distinct disease and should be treated individually and precisely. Further, validation studies are warranted.

\section{DATA AVAILABILITY STATEMENT}

The datasets presented in this study can be found in online repositories. The names of the repository/repositories

\section{REFERENCES}

1. Cicin I, Karagol H, Uzunoglu S, Uygun K, Usta U, Kocak Z, et al. Extrapulmonary small-cell carcinoma compared with small-cell lung carcinoma: a retrospective single-center study. Cancer. (2007) 110:106876. doi: $10.1002 / \mathrm{cncr} .22887$

2. Ishida H, Kasajima A, Onodera Y, Konno T, Maruyama S, Okamoto $\mathrm{H}$, et al. A comparative analysis of clinicopathological factors between esophageal small cell and basaloid squamous cell carcinoma. Medicine. (2019) 98:e14363. doi: 10.1097/MD.0000000000014363

3. Quinn AM, Blackhall F, Wilson G, Danson S, Clamp A, Ashcroft L, et al. Extrapulmonary small cell carcinoma: A clinicopathological study with identification of potential diagnostic mimics. Histopathology. (2012) 61:45464. doi: 10.1111/j.1365-2559.2012.04247.x

4. Liu D, Xu X, Wen J, Xie L, Zhang J, Shen Y, et al. Integrated genome-wide analysis of gene expression and dna copy number variations highlights stem cell-related pathways in small cell esophageal carcinoma. Stem Cells Int. (2018) 2018:1-8. doi: 10.1155/2018/3481783

5. Sohda M, Saeki H, Kuwano H, Miyazaki T, Yokobori T, Sano A, et al. Diagnostic immunostaining and tumor markers predict the prognosis of esophageal neuroendocrine cell carcinoma patients. Ann Surg Oncol. (2021). doi: 10.1245/s10434-021-09872-5. [Epub ahead of print].

6. Verma V, Sleightholm RL, Fang P, Ryckman JM, Lin C. National cancer database report of non-metastatic esophageal small cell and accession number(s) can be found in the article/Supplementary Material.

\section{ETHICS STATEMENT}

Written informed consent was obtained from the individual(s) for the publication of any potentially identifiable images or data included in this article.

\section{AUTHOR CONTRIBUTIONS}

Conception and design: DL, ZZ, and MF. Collection and assembly of data: JW, BW, and JC. Data analysis and interpretation: DL, JW, and XX. All authors contributed to manuscript writing, final approval of manuscript, and accountable for all aspects of the work.

\section{FUNDING}

This study was supported by the Fund for Young Doctor from Shanghai Anticancer Association (Grant No. SACA-CY19C12).

\section{ACKNOWLEDGMENTS}

We thank the American Journal Experts (https://www.aje.com/) for editing this manuscript. The content of this manuscript has been presented in part at the 56th Annual Meeting of the American Society for Radiation Oncology, San Francisco (September 14-17, 2014).

\section{SUPPLEMENTARY MATERIAL}

The Supplementary Material for this article can be found online at: https://www.frontiersin.org/articles/10.3389/fsurg. 2021.655159/full\#supplementary-material

carcinoma. Cancer Med. (2018) 7:6365-73. doi: 10.1002/cam4. 1712

7. Wang F, Liu D-B, Zhao Q, Chen G, Liu X-M, Wang Y-N, et al. The genomic landscape of small cell carcinoma of the esophagus. Cell Res. (2018) 28:7714. doi: 10.1038/s41422-018-0039-1

8. Chen WW, Wang F, Chen S, Wang L, Ren C, Luo HY, et al. Detailed analysis of prognostic factors in primary esophageal small cell carcinoma. Ann Thorac Surg. (2014) 97:1975-81. doi: 10.1016/j.athoracsur.2014. 02.037

9. Purwar P, Jiwnani S, Karimundackal G, Pramesh CS. Management of esophageal small cell carcinoma. Ann Thorac Surg. (2015) 99:1488. doi: 10.1016/j.athoracsur.2014.11.029

10. Hogan BLM, Barkauskas CE, Chapman HA, Epstein JA, Jain R, Hsia CCW, et al. Repair and regeneration of the respiratory system: complexity, plasticity, and mechanisms of lung stem cell function. Cell Stem Cell. (2014) 15:12338. doi: 10.1016/j.stem.2014.07.012

11. Morrisey EE, Rustgi AK. The lung and esophagus: developmental and regenerative overlap. Trends Cell Biol. (2018) 28:73848. doi: 10.1016/j.tcb.2018.04.007

12. Vousden BKH, Yang M. Tissue of origin is important in determining how tumors rewire their metabolism. Science. (2016) 538:397-401. doi: 10.1038/nature19807

13. Cancer Genome Atlas Research Network, Analysis Working Group: Asan University, BC Cancer Agency, Brigham Women's Hospital, Broad Institute, 
Brown University, et al. Integrated genomic characterization of oesophageal carcinoma. Nature. (2017) 541:169-75. doi: 10.1038/nature20805

14. Rudin CM, Durinck S, Stawiski EW, Poirier JT, Modrusan Z, Shames DS, et al. Comprehensive genomic analysis identifies SOX2 as a frequently amplified gene in small-cell lung cancer. Nat Genet. (2012) 44:11116. doi: $10.1038 / \mathrm{ng} .2405$

15. Peifer M, Fernández-Cuesta L, Sos ML, George J, Seidel D, Kasper LH, et al. Integrative genome analyses identify key somatic driver mutations of small-cell lung cancer. Nat Genet. (2012) 44:1104-10. doi: 10.1038/ ng.2396

16. DeRisi J, Penland L, Brown PO, Bittner ML, Meltzer PS, Ray M, et al. Use of a cDNA microarray to analyse gene expression patterns in human cancer. Nat Genet. (1996) 14:457-60. doi: 10.1038/ng1296-457

17. Anthony LB, Strosberg JR, Klimstra DS, Maples WJ, O'Dorisio TM, Warner RRP, et al. The NANETS consensus guidelines for the diagnosis and management of gastrointestinal neuroendocrine tumors (NETs). Pancreas. (2010) 39:767-74. doi: 10.1097/MPA.0b013e3181ec 1261

18. Chow V, Law S, Lam KY, Luk JM, Wong J. Telomerase activity in small cell esophageal carcinoma. Dis Esophagus. (2001) 14:13942. doi: 10.1046/j.1442-2050.2001.00172.x

19. Walenkamp AME, Sonke GS, Sleijfer DT. Clinical and therapeutic aspects of extrapulmonary small cell carcinoma. Cancer Treat Rev. (2009) 35:22836. doi: 10.1016/j.ctrv.2008.10.007

20. Brenner B, Tang LH, Klimstra DS, Kelsen DP. Small-cell carcinomas of the gastrointestinal tract: a review. J Clin Oncol. (2004) 22:27309. doi: 10.1200/JCO.2004.09.075

21. Zhao Q, Chen YX, Wu QN, Zhang C, Liu M, Wang YN, et al. Systematic analysis of the transcriptome in small-cell carcinoma of the oesophagus reveals its immune microenvironment. Clin Transl Immunol. (2020) 9:115. doi: $10.1002 /$ cti2.1173

22. Madroszyk A, Egreteau J, Martin L, Queneau PE, Bosset JF, Merrouche Y. Small-cell carcinoma of the esophagus: report of three cases and review of the literature with emphasis on therapy. Ann Oncol Off J Eur Soc Med Oncol. (2001) 12:1321-5. doi: 10.1023/A:10122217 08372

23. Lv J, Liang J, Wang J, Wang L, He J, Xiao Z, et al. Primary small cell carcinoma of the esophagus. J Thorac Oncol. (2008) 3:14605. doi: 10.1097/JTO.0b013e31818e1247
24. Meng MB, Zaorsky NG, Jiang C, Tian LJ, Wang HH, Liu CL, et al. Radiotherapy and chemotherapy are associated with improved outcomes over surgery and chemotherapy in the management of limited-stage small cell esophageal carcinoma. Radiother Oncol. (2013) 106:317-22. doi: 10.1016/j.radonc.2013.01.008

25. Yun J-P, Zhang M-F, Hou J-H, Tian Q-H, Fu J, Liang X-M, et al. Primary small cell carcinoma of the esophagus: clinicopathological and immunohistochemical features of 21 cases. BMC Cancer. (2007) 7:38. doi: 10.1186/1471-2407-7-38

26. Yamamoto J, Ohshima K, Ikeda S, Iwashita A, Kikuchi M. Primary esophageal small cell carcinoma with concomitant invasive squamous cell carcinoma or carcinoma in situ. Hum Pathol. (2003) 34:110815. doi: 10.1053/j.humpath.2003.07.010

27. Sasajima K, Hayashi N, Yamashita K, Onda M, Takubo K. Oat cell carcinoma of the esophagus with multiple differentiation. J Clin Gastroenterol. (1988) 10:667-71. doi: 10.1097/00004836-1988120 00-00020

28. Frese KK, Simpson KL, Dive C. Small cell lung cancer enters the era of precision medicine. Cancer Cell. (2021) 39:2979. doi: 10.1016/j.ccell.2021.02.002

Conflict of Interest: The authors declare that the research was conducted in the absence of any commercial or financial relationships that could be construed as a potential conflict of interest.

Publisher's Note: All claims expressed in this article are solely those of the authors and do not necessarily represent those of their affiliated organizations, or those of the publisher, the editors and the reviewers. Any product that may be evaluated in this article, or claim that may be made by its manufacturer, is not guaranteed or endorsed by the publisher.

Copyright (C) $2021 \mathrm{Liu}$, Wen, Chen, Wang, Xu, Zhang and Fan. This is an open-access article distributed under the terms of the Creative Commons Attribution License (CC BY). The use, distribution or reproduction in other forums is permitted, provided the original author(s) and the copyright owner(s) are credited and that the original publication in this journal is cited, in accordance with accepted academic practice. No use, distribution or reproduction is permitted which does not comply with these terms. 